\title{
Determination of the gyrotropic characteristics of hexaferrite ceramics from 75 to $600 \mathrm{GHz}$.
}

\author{
Bin Yang, R J Wylde, D H Martin, P Goy, R S Donnan and S Caroopen.
}

\begin{abstract}
The work reported in this paper is in support of applications of ferrites in measurement systems operating at frequencies in the range $\mathbf{1 0 0}$ to $600 \mathrm{GHz}$. Measurements of the magneto-optical characteristics of selected grain-oriented hexaferrite ceramics from 75 to $600 \mathrm{GHz}$ are described; a quasi-optical transmissometer driven by a multi-band vector-network-analyser is used to provide the required high dynamic range, spectral-resolution, and scan-speeds. The implications of the results of the measurements are examined.
\end{abstract}

Index Terms- Uniaxial gyrotropic materials. Hexaferrites. Millimetrewave and submillimetre-wave magneto-optics.

\section{INTRODUCTION}

Recent papers [1] have reviewed the diverse applications of ferrite materials in non-reciprocal devices operating at microwave and millimeter-wave frequencies and have described the improvements in device performance that should come with new methods for fabricating and processing these materials. The work reported in this paper has been undertaken in support of a newly developing area for the application of ferrite-based non-reciprocal devices, viz. in measurement systems operating at frequencies above 100 $\mathrm{GHz}$, into the sub-millimeter-wave range up to $600 \mathrm{GHz}$. Measurement systems operating at such frequencies are now in use in many fields of application, in astronomy and cosmology, in studies of the Earth's atmosphere, in diagnostics of thermo-nuclear plasmas and free-electron lasers, in the characterization of materials, in radar crosssection modeling, in medical and structural imaging. A few such systems at present include non-reciprocal devices (circulators) [2] but there will be many opportunities to deploy such devices in future systems as the efficiencies and bandwidths of these devices in this range of frequency improve. In the search for candidate magnetic materials, and in characterizing samples of the best of them with a precision that will be sufficient for the engineering design of the highperformance circulators that will incorporate them, a method for the rapid determination of the gyrotropic magneto-optical constants of such materials from 75 to $600 \mathrm{GHz}$ has been required. The work reported here demonstrates such a method, employing a quasi-optical transmissometer driven by multiband vector-network-analysers, and identifies a commercially -available hexaferrite ceramic which has been shown, by

Manuscript received September 15, 2009; revised May 31, 2010.

Bin Yang, D H Martin and R. Donnan are at the Dept. of Electronic Engineering, Queen Mary, University of London, and London E1 4NS, UK;

P. Goy and S. Caroopen are at AB Millimetre, Paris and R J Wylde is at Thomas Keating, Billingshurst, UK. measurements using this method, to be well suited for use in wide-band, high-efficiency, circulators to $350 \mathrm{GHz}$ and beyond.

High-performance measurement systems operating at frequencies beyond $100 \mathrm{GHz}$ have open, quasi-optical, structures rather than wave-guiding structures. The magnetic material in a quasi-optical circulator suitable for incorporation in such a system will be in the form of a thin plate magnetized to near-saturation perpendicular to its surfaces, through which the signal beams pass, along its axis. To have a thin plate biased magnetically in this way without an (impracticably) large external magnet to countervail the large demagnetizing field inside the plate, the material in the plate must have excellent permanent-magnet properties with a particularly high ratio of coercivity to saturation magnetization [2]. A metallic permanent-magnet material would, of course, not be suitable in this application but non-metallic hexaferrite ceramic materials with excellent permanent-magnet properties have become commercially-available in recent years and $a \mathrm{few}$ of these meet this demanding criterion [2]. Furthermore, the hexaferrites are known to exhibit magnetic-resonance at frequencies in the millimeter-wave range [3] and this promised that these materials would have well-developed gyrotropic characteristics well into the submillimeter-wave range, a promise confirmed by the measurements reported here.

There had been less confidence that hexaferrite materials would have low submillimeter-wave attenuations. The complicated ionic crystal structures within the grains of these ceramic materials, and the amorphous material binding the grains together, might be expected to support lossy conductive processes at those frequencies, and we have found that to be the case for many of the commercially-available hexaferrite ceramics. However, the measurements we report here have revealed one grain-oriented strontium hexaferrite which does show relatively low attenuation well into the submillimetrewave range (hexaferrite FB6H supplied by TDK [4]). We have been able to use this material in wide-band quasi-optical circulators that are now operating in several major measurement systems [2]. It is not yet wholly clear why this hexaferrite material in particular should have relatively low dielectric losses and further investigation of the compositions and processing used in forming this material will be of interest.

II. THE MAGNETO-OPTICAL CHARACTERISTICS OF A UNIAXIAL GYROTROPIC MATERIAL. 
The permanent-magnet properties, and the submillimeterwave magneto-optical properties, of a grain-oriented hexaferrite ceramic are both attributable to (a) the very large uniaxial magnetocrystalline anisotropy within each grain, (b) the single-domain size of the grains (a few microns) and (c) the high degree of alignment of the hexagonal axes of the grains (a few degrees of spread). Perfect alignment of the grains' axes (a single crystal) would not be helpful in the present context because it would obviate the achievement of high coercivity. With imperfect alignment, a material biased to near-saturation will have random deviations of the spontaneous magnetization from strict uniformity and this lack of optical homogeneity could result in some diffuse scattering of power out of a coherent signal beam propagating in the material. In the grain-oriented hexaferrite ceramics, however, these deviations are of small-amplitude and of very shortrange compared to submillimeter wavelengths; for these reasons it would be expected that diffuse scattering of a submillimeter-wave will be weak. The material would control the propagation of a spatially coherent submillimeter-wave beam essentially as would a homogeneous uniaxial gyrotropic material, but with some attenuation of this coherent beam due to the diffuse scattering. (This is analogous to the well-known division of a signal-beam into spatially-coherent and spatiallyincoherent components on reflection at a metallic surface which has short-range random deviations from a smooth profile, the form of the coherent component being determined by the underlying smooth profile and the spatially-incoherent component being the power diffusely scattered by the random deviations). In a quasi-optical network containing a plate of a gyrotropic ceramic magnetized perpendicular to its surfaces, with a well-collimated beam incident normally on the plate, the spatially-coherent component of the transmitted beam is transferred efficiently to the receiver whilst any diffuselyscattered power will be lost and will be registered at the receiver as an attenuation of the spatially-coherent transmitted beam. This is the model we adopt for a magnetically-biassed fine-grained hexaferrite ceramic that is a uniform uniaxial gyrotropic medium with contributions to the imaginary parts of its propagation constants which represent the weak loss of diffusely-scattered signal-power.

A magnetically-biased homogeneous uniaxial gyrotropic material has a large and uniform spontaneous magnetization, $\mathbf{M}$, which lies preferentially in the direction of the axis. A transverse magnetic field would turn $\mathbf{M}$ away from the axis but the transverse magnetic susceptibility would be of very small magnitude; that is to say, the material would have a large uniaxial magnetic anisotropy. In a wide plate of the material, with $\mathbf{M}$ normal to the faces of the plate, the demagnetizing field, $\mathbf{H}$, would act along the axis. The material then has the uniaxial gyrotropic symmetry characteristic of the axial-vectors $\mathbf{M}$ and $\mathbf{H}$. It is well-known that in such a system circularly-polarised transverse plane-waves propagating in the direction of the axis are characteristic, or normal, modes of the system, that is they propagate independently $[5,6]$. Each of the constituent RF fields in such a wave, $\mathbf{h}(\omega, z, t), \mathbf{b}(\omega, z, t), \mathbf{e}(\omega, z, t)$ and $\mathbf{d}(\omega, z, t)$ where the $z$ axis is in the direction of $\mathbf{M}$ and $\mathbf{H}$, is transverse to the axis and has the following form (as a Jones vector):

$$
\left[\begin{array}{c}
f_{x}(\omega, z, t) \\
f_{y}(\omega, z, t)
\end{array}\right]_{ \pm}=f_{ \pm} \cdot \exp -i \omega\left(t-n_{ \pm}(\omega) \cdot z / c\right) \cdot\left[\begin{array}{c}
1 \\
\pm i
\end{array}\right]
$$

where $\mathbf{f}_{ \pm}(\omega, z, t)$ denotes any of these constituent fields and $f_{ \pm}$ is its complex amplitude. The complex circular-polarisation refractive index, $n_{ \pm}(\omega)$, in the propagation factor, is a characteristic magneto-optical constant of the material equal to $\sqrt{\mu_{ \pm}(\omega) \cdot \varepsilon_{ \pm}(\omega)}$ where $\mu_{ \pm}(\omega)$ is the transverse circularpolarisation permeability of the material and $\varepsilon_{ \pm}(\omega)$ its permittivity. $n_{ \pm}(\omega)$ will be complex if these characteristic plane-waves suffer attenuation in the material.

Waves of both senses of circular-polarization are included here using a notation we shall use throughout. Where a subscript is concerned, the upper alternative $(+)$ denotes the clockwise circular-polarization looking in the direction of $\mathbf{M}$, and the lower $(-)$ denotes the anti-clockwise circularpolarization. Where an in-line sign-alternative (either \pm or $\mp$ ) is concerned, the upper sign is to be taken for the + polarization, and the lower for the - polarization.

The characteristic plane-waves above are those propagating in the positive $z$ direction, parallel to $\mathbf{M}$. There are circularlypolarised plane-waves propagating in the negative $z$ direction, anti-parallel to $\mathbf{M}$, which are also characteristic modes of the material; these have the same values of the refractive indices, $n_{ \pm}(\omega)$, as the former when $+/-$ polarisations again refer to clockwise/anticlockwise rotation looking along $\mathbf{M}$.

To completely specify such a circularly-polarised plane-wave propagating along the axis of a uniaxial gyrotropic material, values for the wave-impedance, $Z_{ \pm}(\omega)$, as well as for the refractive index,$n_{ \pm}(\omega)$, are required. $Z_{ \pm}(\omega)$ is a measure of the ratio of the complex amplitudes of the electric and magnetic fields in the wave. In terms of the underlying transverse circular-polarisation permeabilities and permittivities the reduced wave-impedance, $z_{ \pm}(\omega) \equiv Z_{ \pm}(\omega) / Z_{O}$ where $Z_{O}$ is the free-space impedance, is $[5,6]$

$$
z_{ \pm}(\omega) \equiv Z_{ \pm}(\omega) / Z_{O}=\sqrt{\mu_{ \pm}(\omega) / \varepsilon_{ \pm}(\omega)}
$$

$n_{ \pm}(\omega), z_{ \pm}(\omega)$ and $\mu_{ \pm}(\omega), \varepsilon_{ \pm}(\omega)$ are alternative statements of the magneto-optical characteristics of a uniaxial gyrotropic material. The former are more directly measurable than the latter and are more directly invoked in calculations of the magneto-optical properties of devices that incorporate the material; the latter are more directly involved when seeking insights into the origins of magneto-optical behaviour in the microscopic dynamical responses of a material to transverse RF fields. 
The measurements of $n_{ \pm}(\omega)$ for hexaferrite ceramics which we report here were based on measurements of the circularpolarisation normal-incidence transmittances of a wide plate of the material, with the material axis perpendicular to the faces of the plate. The incident $\mathrm{mm} / \mathrm{submm}$-wave beams in these measurements were much wider than their wavelengths and are therefore effectively plane-waves (see Section III). On entering the plate the beam suffers partial reflections at the surfaces of the plate leading to multiple passes through the thickness of the plate, each pass being a characteristic planewave mode of the material of one circular-polarisation [5]. The circular-polarisation transmittance of the plate, $t_{ \pm}(\omega, d)$, is then $[6,7]$ :

$$
t_{ \pm}(\omega, d)=\frac{\left(1-r_{1 \pm}^{2}(\omega, d)\right) \cdot \exp i \phi_{ \pm}(\omega, d)}{1-r_{1 \pm}^{2}(\omega, d) \cdot \exp i 2 \phi_{ \pm}(\omega, d)}
$$

where $\phi_{ \pm}(\omega, d)$ is the complex angle $\omega \cdot n_{ \pm}(\omega) \cdot d / c$ and $r_{1 \pm}(\omega, d)$ is the single-surface reflectance which is related to the reduced wave-impedance,

$$
r_{1 \pm}(\omega, d) \equiv\left(z_{ \pm}(\omega)-1\right) /\left(z_{ \pm}(\omega)+1\right) .
$$

The determination of the refractive indices, $n_{ \pm}(\omega)$, would be straightforward if reflectances, as well as the transmittances, of the plate-sample were reliably measured; there are complications, however, in making precise measurements of reflectance rapidly and over a wide range of frequency (see Section III) and we have therefore used measured values of transmittances only, following an interpolation procedure explained in Section III.

Moreover, it is not feasible to measure directly the circularpolarisation transmittances over a wide range of frequency at high resolution (see Section III); instead the plate's linearpolarisation transmittances, both co- and cross-polar, $t_{c o}(\omega, d)$ and $t_{c r}(\omega, d)$ respectively, are first measured and the results then transformed into $t_{ \pm}(\omega, d)$ thus:

$$
t_{ \pm}(\omega, d) \equiv t_{c o}(\omega, d) \mp i t_{c r}(\omega, d)
$$

These relationships are established as follows. A $2 \times 2$ transmittance matrix operates on the Jones vector of a signal beam of arbitrary polarization-state incident on a sample plate to determine the Jones vector of the emergent beam. For a gyrotropic plate this matrix has the form:

$$
\left[\begin{array}{cc}
t_{c o}(\omega, d) & -t_{c r}(\omega, d) \\
t_{c r}(\omega, d) & t_{c o}(\omega, d)
\end{array}\right]
$$

as can be confirmed by noting that, when this matrix operates on a circularly-polarised incident beam, it generates, as is required by boundary conditions, a circularly-polarised transmitted beam of the same sense, thus

$$
\begin{aligned}
& {\left[\begin{array}{ll}
t_{c o}(\omega, d) & -t_{c r}(\omega, d) \\
t_{c r}(\omega, d) & t_{c o}(\omega, d)
\end{array}\right]\left[\begin{array}{c}
1 \\
\pm i
\end{array}\right]=} \\
& \left(t_{c o}(\omega, d) \mp i t_{c r}(\omega, d)\right)\left[\begin{array}{c}
1 \\
\pm i
\end{array}\right] \equiv t_{ \pm}(\omega, d)\left[\begin{array}{c}
1 \\
\pm i
\end{array}\right]
\end{aligned}
$$

which provides the relationships above. The elements of the matrix, $t_{c o}(\omega)$ and $t_{c r}(\omega)$, can be identified as the co-polar and cross-polar linear-polarisation transmittances since:

$$
\begin{gathered}
{\left[\begin{array}{cc}
t_{c o} & -t_{c r} \\
t_{c r} & t_{c o}
\end{array}\right]\left[\begin{array}{l}
1 \\
0
\end{array}\right]=t_{c o}\left[\begin{array}{l}
1 \\
0
\end{array}\right]+t_{c r}\left[\begin{array}{l}
0 \\
1
\end{array}\right] \text { and }} \\
{\left[\begin{array}{cc}
t_{c o} & -t_{c r} \\
t_{c r} & t_{c o}
\end{array}\right]\left[\begin{array}{l}
0 \\
1
\end{array}\right]=t_{c o}\left[\begin{array}{l}
0 \\
1
\end{array}\right]-t_{c r}\left[\begin{array}{l}
1 \\
0
\end{array}\right]}
\end{gathered}
$$

The intrinsic symmetries of a uniaxial gyrotropic material determine that the characteristic plane-wave modes propagating along the axis are circularly-polarised but the values of the refractive indices and wave-impedances of these waves, $n_{ \pm}(\omega)$ and $z_{ \pm}(\omega)$, are determined by the detail of the local dynamical responses of the material to transverse circularly-polarised time-harmonic fields. Measured values of $n_{ \pm}(\omega)$ and $z_{ \pm}(\omega)$ for a given material can be used in designing a device containing that material without knowing the nature of those dynamical processes. Consideration of the dynamical processes that might be involved can, however, give guidance on what values and frequency-dependences of $n_{ \pm}(\omega)$ and $z_{ \pm}(\omega)$ might be expected. For this reference is made to the local transverse circular-polarisation permeabilities and permittivities of the material, $\mu_{ \pm}(\omega)$ and $\varepsilon_{ \pm}(\omega)$, which are related to $n_{ \pm}(\omega)$ and $z_{ \pm}(\omega)$ thus [5,6]:

$$
n_{ \pm}=\sqrt{\mu_{ \pm}(\omega) \cdot \varepsilon_{ \pm}(\omega)} ; z_{ \pm}(\omega)=\sqrt{\mu_{ \pm}(\omega) / \varepsilon_{ \pm}(\omega)}
$$

There is a well-known simple dynamical model for the transverse permeability of a uniaxial gyrotropic material [5]. The spontaneous magnetization $\mathbf{M}$ in the material experiences a restoring couple $\mathbf{M} \times\left(\mathbf{H}_{a}+\mathbf{H}_{d}\right)$ when it deviates transversely from the axis, where $\mathbf{H}_{a}$ is an effective field representing the uniaxial magnetocrystalline anisotropy of the material and $\mathbf{H}_{d}$ is a uniform magnetic field in the material acting along the axis. Since $\mathbf{M}$ is intrinsically associated with angular momentum (that of the electrons that give rise to $\mathbf{M}$ ) it responds gyroscopically to a transverse circular-polarisation magnetic field, $h_{ \pm}(\omega)$, i.e. it undergoes precessional motion and thereby generates a transverse magnetic induction $b_{ \pm}(\omega)=\mu_{ \pm}(\omega) \cdot h_{ \pm}(\omega)$, with $\mu_{ \pm}(\omega)$ depending on frequency:

$$
\mu_{ \pm}(\omega)=1+\frac{\omega_{m}}{\omega_{0} \mp \omega}
$$


where $\omega_{0} \equiv|\gamma|\left(H_{a}+H_{d}\right), \omega_{m} \equiv|\gamma| M$, and the parameter $\gamma$ is the ratio of the magnetic moment of an electron in the material to its associated angular momentum, which is close in value to $\mu_{0} e / \mathrm{m}$, where $\mathrm{e} / \mathrm{m}$ is the charge-to-mass ratio for a free electron. It can be seen that the permeability for the + circular-polarisation is resonant at $\omega_{0}$ whereas the permeability for - circular-polarisation is not resonant; this is a consequence of the fact that free precessional motion of $\mathbf{M}$ is of + sense. If $\mathbf{H}_{d}$ is the demagnetising field in a thin plate magnetised normally to its surfaces, $\mathbf{H}_{d}=-\mathbf{M}$ and the resonance frequency is then $\omega_{0}=\omega_{a}-\omega_{m}$ where $\omega_{a} \equiv|\gamma| H_{a}$. Damping of the precession would contribute an imaginary part to the permeability.

Weakly-damped magnetic resonance of this kind has been observed in grain-oriented strontium hexaferrite ceramics at frequencies in the millimetre-wave range [3] which correspond well with directly measured values of the static anisotropy fields and spontaneous magnetisations of these materials. Our measurements of the refractive indices for a selected strontium hexaferrite to frequencies well beyond the resonance are also consistent with this simple dynamical model (Section III).

Finding a model for the circular-polarisation transverse permittivity of a hexaferrite material at submillimeter-wave frequencies is less straightforward. A hexaferrite is an ionic crystalline material and as such has optically-active lattice vibrations with resonance frequencies above the submillimeter-wave range, in the far-infrared [8]; the driving of those vibrations at submillimeter-wave frequencies makes a contribution to the real parts of the material's permittivities at those frequencies, a contribution that would be expected to increase relatively slowly with frequency through that range since these frequencies are well below the resonance frequencies of the optically-active lattice vibrations. Damping of these driven lattice vibrations makes a contribution to the imaginary part of the permittivity and this would be expected to increase with frequency in this range from a low initial value. A hexaferrite ceramic is not a single crystal, however, but is structurally complicated, with micron-sized crystalline grains bonded by amorphous inter-grain material, and perhaps also with some disorder in the complex lattice-cells within the grains. Such structural defects would give some optical activity to low frequency lattice vibrations that would be optically inactive in a perfect lattice. This will make a small contribution to the real part of the permittivity and a contribution to the imaginary part that would be expected to increase strongly with frequency through the submillimeterwave range. The differences between the permittivities for the two senses of circular-polarisation are usually implicitly assumed to be negligible in the millimetre-wave range, a material's gyrotropic properties being attributed to differences in the permeabilities for the two senses, as indicated above. (At very much higher frequencies, in the infrared and visible spectra, the magneto-optical behaviour of magnetic materials is known to arise from differences in the permittivities for the two senses of circular-polarisation associated with electronic transitions or resonances). We have already remarked on the diffuse scattering of power out of a coherently-propagating beam at magnetic inhomogeneities in a ceramic material, and the contribution which that might make to the imaginary parts of both the permeability and the permittivity; this contribution too would be expected to increase with frequency.

The results of our measurements of $n_{ \pm}(\omega)$ for a selected strontium hexaferrite ceramic are discussed in Section III with reference to these model expectations for $\mu_{ \pm}(\omega)$ and $\varepsilon_{ \pm}(\omega)$.

\section{MEASUREMENT OF THE CIRCULAR- POLARISATION REFRACTIVE INDICES OF A HEXAFERRITE CERAMIC FROM 75 TO 600 GHz.}

The samples under study in the measurements reported here were all square plates of the strontium hexaferrite grainoriented ceramic FB6H supplied by TDK [4], of thickness $2.02 \mathrm{~mm}$ and edge-length $100 \mathrm{~mm}$, with the material's axis perpendicular to the plates' faces. Each sample-plate had been magnetized to saturation in the close-fitting pole-gap of a high-field electromagnet and then removed progressively from the electromagnet in a controlled reduction of the poling field, to leave the plate uniformly and stably magnetized to nearsaturation along its axis [2]. Measurements were made of the normal-incidence plane-wave transmittances of these plates at laboratory ambient temperature, nominally $20^{\circ} \mathrm{C}$, over the range 75 to $110 \mathrm{GHz}$ for twenty such plates, and over the range 75 to $600 \mathrm{GHz}$ for one of these. The results were analysed to determine the complex refractive indices of FB6H over that range of frequency.

The quasi-optical transmissometer used for these measurements is illustrated in Fig.1. It is driven by a vectornetwork-analyser (VNA) in each of five successive waveguide bands making-up the range from 75 to $600 \mathrm{GHz}$. The VNA's head-units for each wave-guide band include shaped corrugated feed-horns to transform rectangular-wave-guide modes into linearly-polarised free-space Gaussian beammodes. They were mounted in turn on the transmissometer's base-plate (at H1 and H2 in Fig. 1). The quasi-optical circuit of the transmissometer serves for all five frequency-bands. An ellipsoidal reflector, $\mathrm{F} 1$, receives the signal-beam diverging from the VNA head-unit $\mathrm{H} 1$ and transforms it into a linearlypolarised quasi-collimated beam which passes through the sample-plate at S. A similar ellipsoidal reflector, F2, condenses the transmitted beam into the feed-horn of the VNA head-unit, H2. The shaped, corrugated feed-horns in the VNA head-units, and the ellipsoidal reflectors, are dimensioned so that the signal beam has a Gaussian beamwaist at the sample location, S, with a width-parameter close to $50 \mathrm{~mm}$ throughout each band $[9,10]$. The plane-wave angular-spectrum of the signal beam incident on the sample is therefore Gaussian in form with a width-parameter ranging from 1 degree at $70 \mathrm{GHz}$ to 0.1 degrees at $600 \mathrm{GHz}$, and appreciably smaller than this as the beam refracts into the 
sample. These small angular-widths ensure that the transmissometer essentially measures a sample-plate's planewave transmittance spectra. The sample-plate's width (100 $\mathrm{mm}$ ) is large enough that the beam is negligibly truncated at the edges of the plate. Planar wire-grid polarisers are included in the quasi-optical circuit (G1 and G2) so that measurements can be made of the sample-plate's linear-polarisation transmittances, $\mathrm{CO}$ - and cross-polar. For measurement of $\mathrm{CO}$ polar transmittances, the VNA head-units $\mathrm{H} 1$ and $\mathrm{H} 2$ are both set to transmit/receive vertical polarization, and the wire-grids G1 and G2 are set to reflect, through 90 degrees, the component of an incident beam having vertical polarization (any orthogonally-polarised component being transmitted through the grid and deposited in a planar RAM power-dump, D in Fig.1). For measurement of cross-polar transmittances, the setting of wire-grid G2 is changed to reflect horizontal polarization and the VNA head-unit $\mathrm{H} 2$ is rotated to receive horizontal polarization. The plane reflectors $\mathrm{C}$ in Fig. 1 are quasi-optical directional-couplers each being a dielectric film having a reflectance for the required polarisation of about -8 $\mathrm{dB}$ and the transmitted signal-power being taken up in a planar RAM power-dump, D; for the lower frequency-bands these directional-couplers are not required (see below) and are replaced by planar metal reflectors.

This is an efficiently-coupled transmit/receive system and any reflections at impedance discontinuities within it would give rise to standing-waves which would corrupt the measurements of the transmittance spectra. Computational time-gating functions were available in the VNA for each frequency-band, to filter-out any short-period standing-waves, but this function had to be exercised with care because the total effective pathlength for multiple reflections within a low-loss sample could approach the path lengths between the components of the transmissometer. Care had therefore been taken in the engineering design of the feed-horns in the VNA head-units, which transform rectangular-wave-guide modes into freespace Gaussian beam-modes, to minimize impedance discontinuities [10]. The VNA head-units for the lower frequency-bands included efficient wide-band in-wave-guide isolators, and these were effective in suppressing standing waves. Good in-wave-guide isolators were not available for the higher frequency-bands, however, (in fact, our major purpose in the work reported here was to promote the development of isolators for these higher frequencies); for these bands it was necessary to include the quasi-optical directional-couplers in the transmissometer to dampen out standing waves, though this damping could not be too strongly imposed because it reduced the system's dynamicrange. For the bands above $350 \mathrm{GHz}$ the residual standing waves were efficiently suppressed by off-setting the sample plate by a small angle from exact normal-incidence thereby diverting signal-power reflected from the sample out of the system. An off-set angle greater than the angular-width of the signal-beam's plane-wave spectrum at the sample is required for this; we used a 2 degree off-set angle which, given the large electrical width of the beam in our transmissometer, was more than sufficient for this purpose. For an angle of incidence as small as this, the transmittance of the plate differs negligibly from that for normal incidence [11], and the associated very small lateral displacement of the transmitted beam has negligible effect on the signal-power received by the VNA-head.

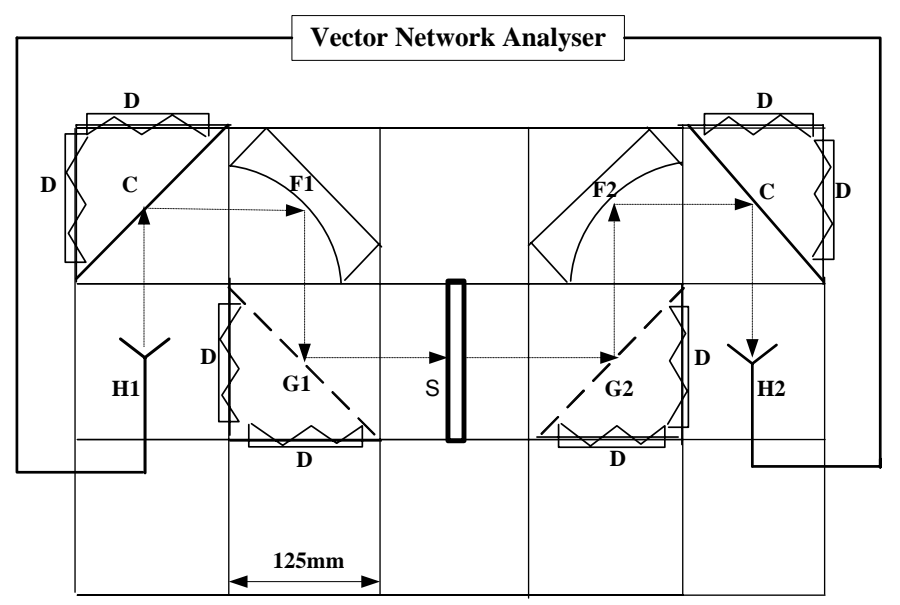

Fig.1. Schematic diagram of the QO transmissometer. $\mathrm{H}$ denotes a corrugated feed-horn, $\mathrm{F}$ an ellipsoidal reflector, $\mathrm{S}$ the ferrite plate under test, $\mathrm{C}$ denotes a quasi-optical directional coupler, $\mathrm{G}$ a wire-grid polarizer and $\mathrm{D}$ power-dumps. The focal distance of each ellipsoidal reflector is $600 \mathrm{~mm}$.

The transmissometer and the feed-horns in the VNA headunits for each of the five frequency-bands were manufactured at Thomas Keating Ltd., UK [10]. The wire-grid polarisers were wound on frames ground flat to $10 \mu \mathrm{m}$, with wire of $10 \mu \mathrm{m}$ diameter. Such grids show cross-polar leakage at the $-50 \mathrm{~dB}$ level in the 100 to $300 \mathrm{GHz}$ range. The planar powerdumps are carbon-loaded polymer pyramid-arrays. They have monostatic reflectances less than $-40 \mathrm{~dB}$. The several components are incorporated in half-cube modules which are configured with high precision on a drilled base-plate.

The procedure followed to determine the co- and cross-polar transmittance spectra of a plate-sample in each frequencyband was as follows. The VNA was used to measure the coand cross-polar insertion losses of the plate by recording the ratios of the complex amplitudes of the transmitted and incident signal-beams (a) with the plate-sample in place, (b) with the plate-sample removed, and (c) with a wire-grid set to transmit $45^{\circ}$ polarization in the place of the plate-sample, in each case for the co-polar and cross-polar settings of the wiregrids and VNA head-units. The measurements (c) are introduced to circumvent any slippage in amplitude or phase incurred on rotating the receiver VNA head-unit from the coto cross-polar settings. If we denote these measured complex ratios by $R c o_{a}, R c o_{b}, R c o_{c}$ respectively for the co-polar cases, and by $R c r_{a}, R c r_{b}, R c r_{c}$ respectively for the cross-polar cases, the complex insertion losses, $L c o$ and $L c r$, are

$$
\begin{aligned}
& L c o=\left(R c o_{a} / R c o_{b}\right) \quad \text { and } \\
& L c r=\left(R c r_{a} / R c r_{C}\right) \cdot\left(R c O_{c} / R c o_{b}\right)
\end{aligned}
$$


and, since each bracketed term here involves only cross-polar ratios or only co-polar ratios, the results for $L c o$ and $L c r$ will not be corrupted by such amplitude or phase slippage.

When the insertion-loss spectra have been measured in this way they are converted to transmittance-spectra by adding the free-space phase-delay, $\omega . d / c$, to the phase at each frequency, where $d$ is the thickness of the sample-plate.

And finally the co- and cross-polar transmittances are transformed into circular-polarisation transmittances using the conversion relations established in Section II.

Ideally, measurements would be made not only of the transmittance spectra of a plate-sample but also of its reflectance spectra. The modules on the base-plate of the transmissometer can in fact be reconfigured to serve as a reflectometer, and we have made some measurements of the reflectances of plate-samples in the lower frequency-bands. However, there are several complications in making reliable reflectance measurements, particularly in the higher frequency-bands. In a reflectance measurement the locations of the plate-sample and of the reference reflector must be determined with high precision to avoid errors in the measurements of the phases, and the orientations and flatnesses of the plate-samples, and of the reference reflectors, must be highly precise in order to avoid errors in the measured amplitudes. Moreover, it is not possible in a reflectance measurement to off-set the sample from exact normal incidence to suppress standing waves. Dealing with these complications adds appreciably to the time taken to make reflectance measurements over a wide range of frequency and, since our objective in this work has been to make rapid and routine determinations of the refractive indices of gyrotropic materials over a wide range of frequency in support of exploratory assessments of many candidate materials, we have followed a procedure for determining refractive indices from measured transmittance spectra only, as explained below.

To check the performance of the transmissometer we first made measurements of the normal-incidence co-polar transmittance spectra of a square plate of high-purity monocrystalline silicon (resistivity $3000 \mathrm{ohm}-\mathrm{cm}, 1.98 \mathrm{~mm}$ thickness and $100 \mathrm{~mm}$ edge-length) through five successive wave-guide bands, from 75 to $600 \mathrm{GHz}$, for which we required the multi-band $\mathrm{AB}$ Millimetre VNA, type MVNA [12]. Fig.2 (a) illustrates the results of these measurements for the four bands from 110 to $600 \mathrm{GHz}$. The plotting routine for this Figure generated the breaks in the lines where the slope is high; to illustrate more clearly the quality of the recorded data, the cycle in this transmittance spectrum at 290 to $330 \mathrm{GHz}$ is separately shown in Fig.2 (b) and that at 500 to $525 \mathrm{GHz}$ in Fig. 2(c).
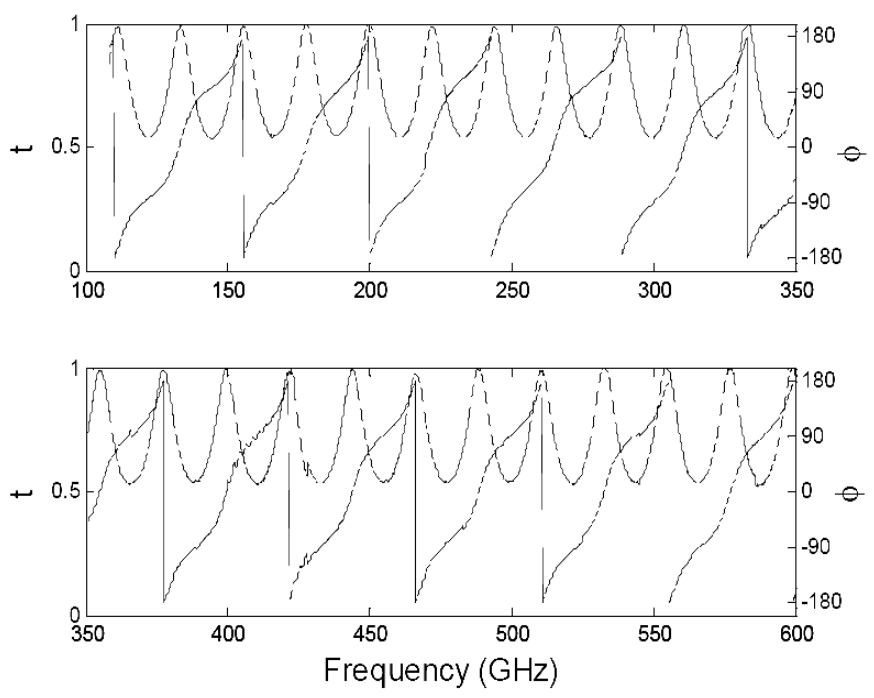

Fig. 2(a). To illustrate the measured complex transmittance spectra of the silicon sample-plate from 110 to $600 \mathrm{GHz}$; t is the modulus of the transmittance and $\Phi$ is its phase-angle in degrees reduced to the zone from +180 to -180 degrees. The broken form of the lines in this Figure is an artefact of the plotting routine at large slopes; it does not represent discontinuities in the recorded data (see Figures $2 \mathrm{~b}$ and 2c). The recorded data are at $10 \mathrm{MHz}$ resolution.

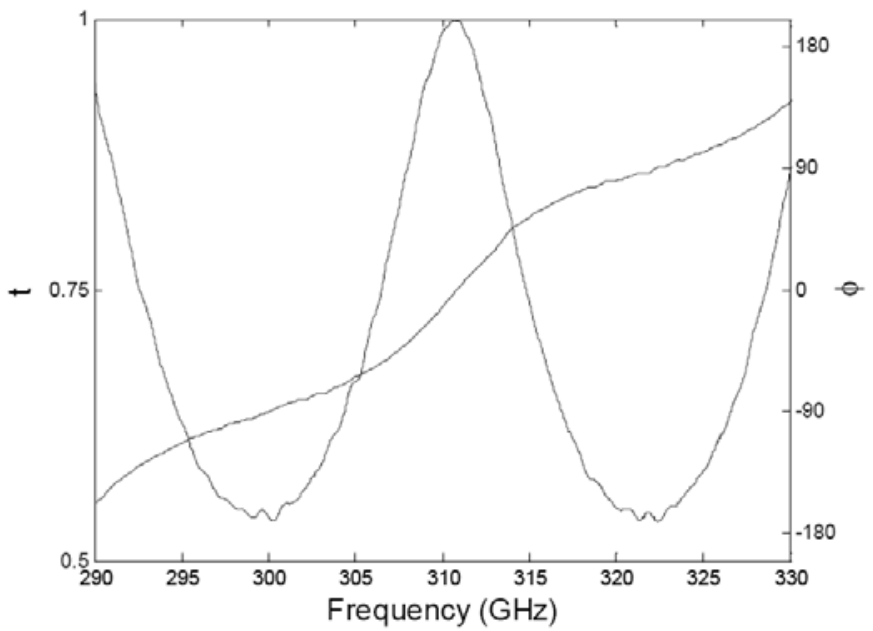

Fig. 2(b). To illustrate the measurements of the complex transmittance spectra of the silicon sample-plate from 290 to $300 \mathrm{GHz}$; $t$ is the modulus of the transmittance and $\Phi$ is its phase-angle in degrees.

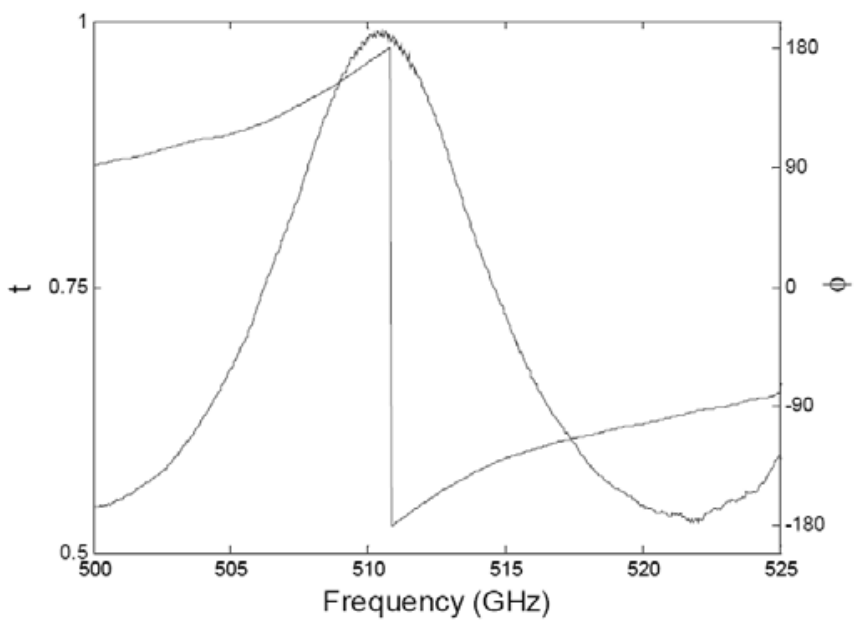

Fig. 2(c). To illustrate the measurements of the complex transmittance spectra 
of the silicon sample-plate from 500 to $525 \mathrm{GHz}$; t is the modulus of the transmittance and $\Phi$ is its phase-angle in degrees.

Measurement of the cross-polar transmittance showed this to be less than $-40 \mathrm{~dB}$ throughout this frequency-range, as is to be expected for an isotropic dielectric material, confirming the control of polarization provided by the wire-grids over this range of frequency.

The periodic character of the transmittance spectrum for silicon in Figure 2(a) is a consequence of interference between multiple passes of the beam within the plate. The high values at the peaks indicate very low loss in this material. The values of the frequencies at the maxima and minima in transmittance serve to determine values for the refractive indices of the silicon at those frequencies. The values obtained in this way for the fifty $\mathrm{max} / \mathrm{min}$ frequencies in the range $100-600 \mathrm{GHz}$ have a mean value of 3.413 with an rms-deviation of 0.004 $(11.65+/-0.02$ for the permittivity) which is very close to that found in other recently reported high-precision measurements on high-purity silicon in this range of frequency [13].

These measurements on a silicon sample-plate serve to illustrate the capability of a quasi-optical transmissometer used in conjunction with a multi-band VNA in the $\mathrm{mm} /$ submillimeter-wave range. This frequency-domain technique provides faster scan-speeds, higher frequencyresolutions, with more nearly plane-wave test-beams, than can be achieved in this range of frequency using time-domain spectrometric techniques [14].

The transmissometer was then used to measure the co-polar and cross-polar transmittances of twenty sample-plates of TDK FB6H hexaferrite ceramic [3], each of thickness 2.02 $\mathrm{mm}$, over the range 75 to $110 \mathrm{GHz}$ using an HP 8510C VNA following the procedures described above [15]. Fig. 3 illustrates the results for one of these sample-plates.

The very deep minimum in the co-polar transmittance at 78.3 $\mathrm{GHz}$ implies that, at that frequency, a signal beam suffers a Faraday rotation of 90 degrees in each forward and backward pass of the $2.02 \mathrm{~mm}$ plate. This serves as a valuable check in the determination of the refractive-index spectra of the material of the plate (see below).
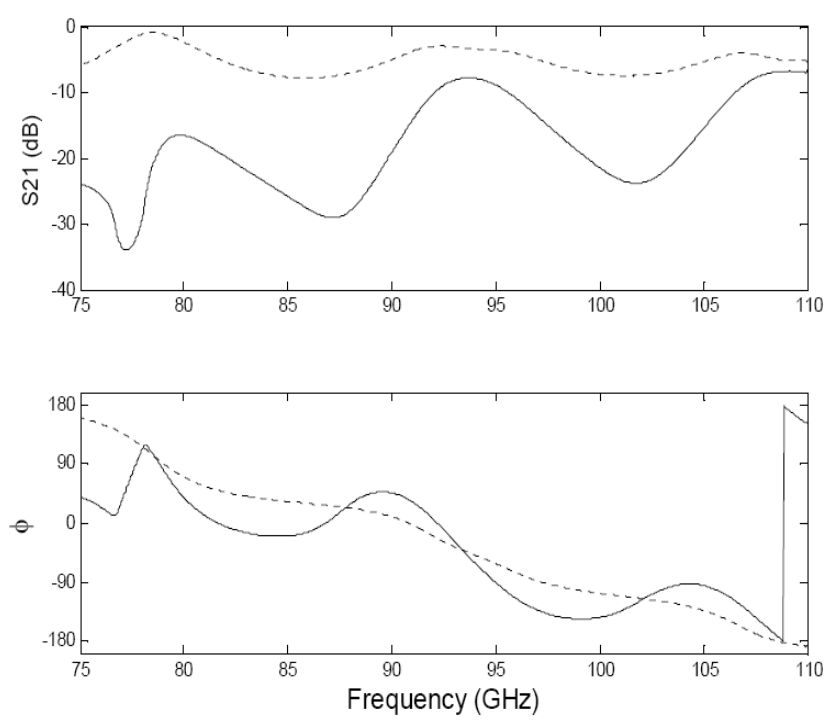

Fig. 3 The measured transmittances of a FB6H hexaferrite plate from 75 to $110 \mathrm{GHz}$ for both the co-polar case (solid line) and the cross-polar case (dotted line), the modulus in $\mathrm{dB}$ above and the phase-angle in degrees below.

The forms of these co- and cross-polar complex-transmittance spectra are complicated and it would not be feasible to analyse them directly to determine the values of the material's circular-polarisation refractive indices. They were therefore transformed into circular-polarisation complex-transmittances as described above; the results are shown in Fig. 4 and the much simpler forms, amenable to analysis, are clear.
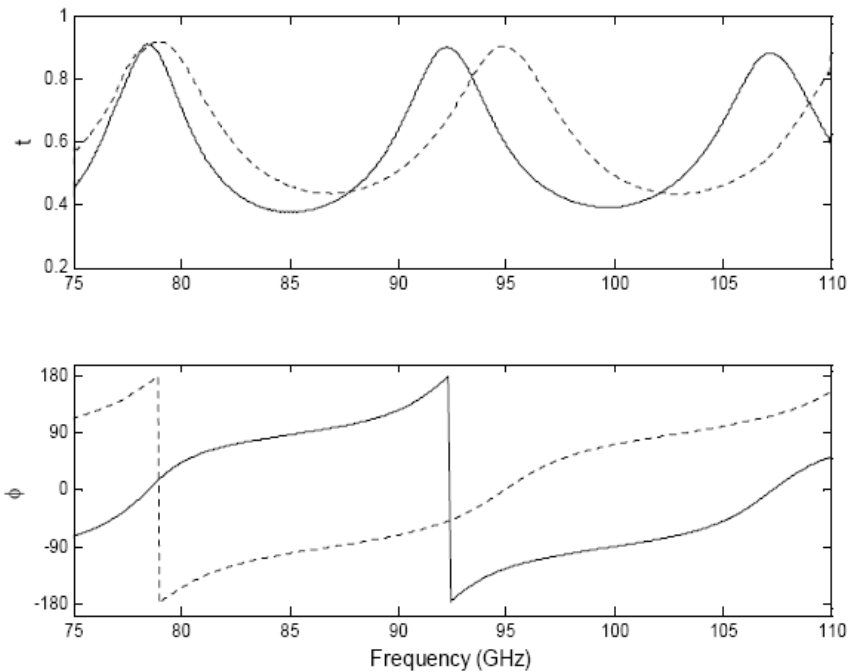

Fig. 4 The measured complex circular-polarisation transmittances of a FB6H ferrite plate from 75 to $110 \mathrm{GHz}$; the solid line is for the + sense of circular polarization and the dotted line is for the - sense, the modulus above and the phase-angle in degrees below.

Small differences were found in the transmittance spectra of the twenty sample-plates of FB6H implying variations in the real part of the refractive indices from one sample-plate to another of a few per cent, and variations in the beamattenuation ranging over a factor of two. Such variations, though small, would be significant for the dimensioning of a non-reciprocal device incorporating a plate of this material. It is clear that, when designing such a device, it will be 
necessary first to have measurements of the optical constants of the particular sample of the material that is to be incorporated in the device.

We selected from this batch of twenty FB6H sample-plates the one that had the least attenuation at $100 \mathrm{GHz}$ (which we label FB6H.V) and we measured its normal-incidence co- and cross-polar complex transmittances through the five waveguide-bands from 75 to $600 \mathrm{GHz}$ using the $\mathrm{AB}$ Millimetre MVNA [12]. These co- and cross-polar transmittances were transformed into complex circularpolarization transmittances, as described above, and the results for each sense are illustrated in Fig. 5.
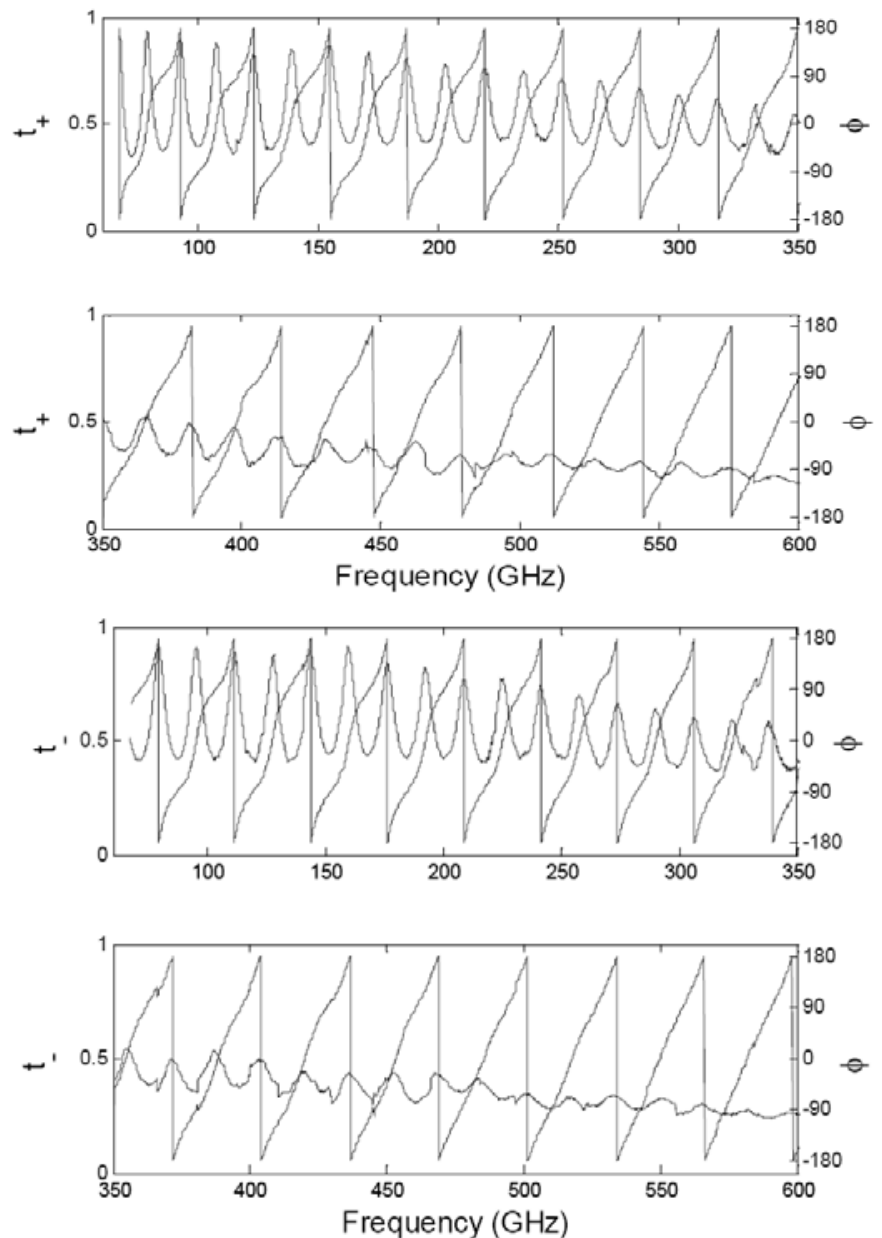

Fig.5. To illustrate the measured complex transmittance spectra of the FB6H.V hexaferrite sample-plate from 75 to $600 \mathrm{GHz}$, for the + sense of circular polarization (upper plots) and for the - sense (lower plots). (The broken form of the lines here is an artefact of the plotting routine at large slopes; it does not represent discontinuity in the recorded data which are at 10 $\mathrm{MHz}$ resolution).

The periodic structure in these transmittance spectra is attributable to interference between multiple-passes of the signal-beam within the plate. The reducing amplitude of the periodic excursions with increasing frequency indicates increasing signal-beam attenuation. The following formula (from Section II) gives the transmittances of the plate, $t_{ \pm}(\omega)$, in terms of the complex refractive indices of the material in the plate, $n_{ \pm}(\omega) \equiv n_{ \pm}^{\prime}(\omega)+i . n_{ \pm}^{\prime \prime}(\omega)$ :

$$
\begin{aligned}
& t_{ \pm}(\omega, d)= \\
& \frac{\left(1-r_{1 \pm}^{2}\right) \cdot \exp \left\{-\omega \cdot n_{ \pm}^{\prime \prime}(\omega) \cdot d / c\right\} \cdot \exp \left\{i \omega \cdot n_{ \pm}^{\prime}(\omega) \cdot d / c\right\}}{1-r_{1 \pm}^{2} \cdot \exp \left\{-2 \omega \cdot n_{ \pm}^{\prime \prime}(\omega) \cdot d / c\right\} \cdot \exp \left\{i 2 \omega \cdot n_{ \pm}^{\prime}(\omega) \cdot d / c\right\}}
\end{aligned}
$$

where $d$ is the thickness of the plate and $r_{1 \pm}(\omega)$ is the singlesurface reflectance. The phase angle of $r_{1 \pm}(\omega)$ is very small provided the beam attenuation coefficient $\omega . n_{ \pm}^{\prime \prime}(\omega) / c$ is not great and it is the factor $\exp \left\{i \omega \cdot n_{ \pm}^{\prime}(\omega) . d / c\right\}$ in this expression that gives $t_{ \pm}(\omega, d)$ its periodic form. The modulus of $t_{ \pm}(\omega, d)$ therefore has local maxima at frequencies $\omega_{N}=N . \pi c / n_{ \pm}^{\prime}\left(\omega_{N}\right) . d$ where $N$ is an integer, and the phase of $t_{ \pm}(\omega, d)$ takes the value 0 (even $N$ ) or $\pm \pi$ (odd $N$ ) at those frequencies. The values of the $\omega_{N}$ can therefore be identified in the data and, once the value of the integer $N$ for any one of these maxima is determined (see below), the values of $N$ at all other $\omega_{N}$ are decided by counting successive maxima. The values of the frequencies $\omega_{N}$ then serve to determine the real part of the refractive index since $n_{ \pm}^{\prime}\left(\omega_{N}\right)=N . \pi c / \omega_{N} . d$. To determine the value of $N$ for a particular maximum in the transmittance spectrum for - ve circular-polarization, that at frequency $\omega_{N^{\prime}}$ say, can be identified as the integer nearest to the value of $2 \omega_{N^{\prime}} /\left(\omega_{N^{\prime}+1}-\omega_{N^{\prime}-1}\right)$ since the rate of change with frequency of the real part of the refractive index for this sense of polarization is expected to be relatively low. A different approach is required for identifying the value of $N$ at a particular maximum in the transmittance spectrum for + circular-polarization because the rate of change with frequency of the real part of the refractive index for this case is expected to be significant because magnetic resonance occurs in this polarization. It is possible to invoke the observation of an extremely low value of the measured copolar linear-polarization transmittance of the plate at 78.3 GHz (Fig. 3) because it implies that a linearly-polarised beam at that frequency suffers a $\pi / 2$ Faraday rotation of its polarization in each forward, and in each backward, traverse of the thickness of the plate. This means that $n_{+}^{\prime}\left(\omega_{F}\right)-n_{-}^{\prime}\left(\omega_{F}\right)=\left(c / \omega_{F} d\right) \pi$, where $\omega_{F}=2 \pi .78 .3 .10^{9}$ and, since the value of $n_{-}^{\prime}\left(\omega_{F}\right)$ is known, as above, this serves to determine the value of $n_{+}^{\prime}\left(\omega_{F}\right)$ from which the values for $N$ at the maxima in the + circular-polarisation transmittance spectrum above and below $\omega_{F}$ can be deduced, and thence values for $N$ and for $n_{+}^{\prime}\left(\omega_{N}\right)$ at all other $\omega_{N}$.

The values obtained in these ways for $n_{-}^{\prime}(\omega)$ and for $n_{+}^{\prime}(\omega)$ at the frequencies of the transmittance maxima are shown in Fig. 6. Corresponding procedures can be followed to determine the values of $n_{ \pm}^{\prime}(\omega)$ at the frequencies of the minima in $t_{ \pm}(\omega, d)$, and these, too, are included in the Figure. 


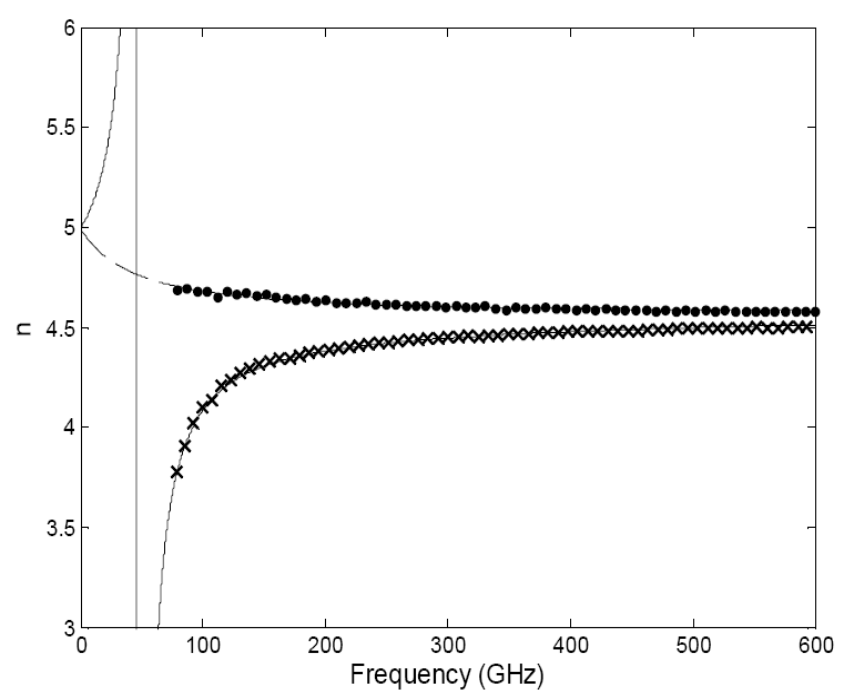

Fig.6. The points in this figure are the measured values of the real part of the circular-polarisation refractive indices for the FB6H.V material (crosses for the + polarization and dots for the - sense). The lines do not represent these measured values but the values obtained using the magnetic resonance model (see the text).

These determinations of the real parts of the refractive indices have required only the frequencies at which the maxima and minima occur. To determine the values for the imaginary parts of the refractive indices, $n^{\prime \prime}\left(\omega_{N}\right)$, the values of maximum transmittance at those frequencies are required. The procedure for this is as follows (the \pm subscripts on the symbols indicating senses of circular polarization can be omitted here since the procedure is to be conducted for each sense of circular polarisation independently. The formula for the transmittance above shows that the modulus of the transmittance at a maximum, at frequency $\omega_{N}$ say, is the value taken at $\omega_{N}$ by the quantity

$$
\frac{\left(1-a(\omega)^{2}\right) \cdot b(\omega, d)}{1-a(\omega)^{2} \cdot b^{2}(\omega, d)}
$$

where $b(\omega, d) \equiv \exp -\left(n^{\prime \prime}(\omega) \cdot \omega \cdot d / c\right)$ and $a(\omega)=\left|r_{1}(\omega)\right|$.

Similarly, the modulus of the transmittance at a minimum is equal to the value of the quantity

$$
\frac{\left(1-a(\omega)^{2}\right) \cdot b(\omega, d)}{1+a(\omega)^{2} \cdot b^{2}(\omega, d)}
$$

at the frequency of that minimum. We now take the value of this latter quantity at the frequency $\omega_{N}$ to be given by a linear interpolation between the values of this quantity at the minima immediately above and below $\omega_{N}$, i.e. a weighted mean of those modules, $\left|\bar{t}\left(\omega_{N}, d\right)\right|$ say. We have, therefore, simultaneous equations relating to the frequency $\omega_{N}$ :

$$
\begin{gathered}
\left|t\left(\omega_{N}, d\right)\right|=\frac{\left(1-a\left(\omega_{N}\right)^{2}\right) b\left(\omega_{N}, d\right)}{1-a\left(\omega_{N}\right)^{2} b\left(\omega_{N}, d\right)^{2}} . \\
\left|\bar{t}\left(\omega_{N}, d\right)\right| \equiv \frac{\left(1-a\left(\omega_{N}\right)^{2}\right) \cdot b\left(\omega_{N}, d\right)}{1+a\left(\omega_{N}\right)^{2} \cdot b\left(\omega_{N}, d\right)^{2}} .
\end{gathered}
$$

Where the values of the quantities on the left are measured values. Eliminating $a\left(\omega_{N}\right)$ from these simultaneous equations then determines a value for $b\left(\omega_{N}, d\right)$ and thence values for the imaginary part of the refractive index $n^{\prime \prime}\left(\omega_{N}\right)$. Values obtained in this way for $\beta(\omega) \equiv(\omega / c) \cdot n^{\prime \prime}(\omega)$, the attenuation coefficient, at the frequency of each transmittance maximum, in each circular-polarisation, are plotted in Fig.7.

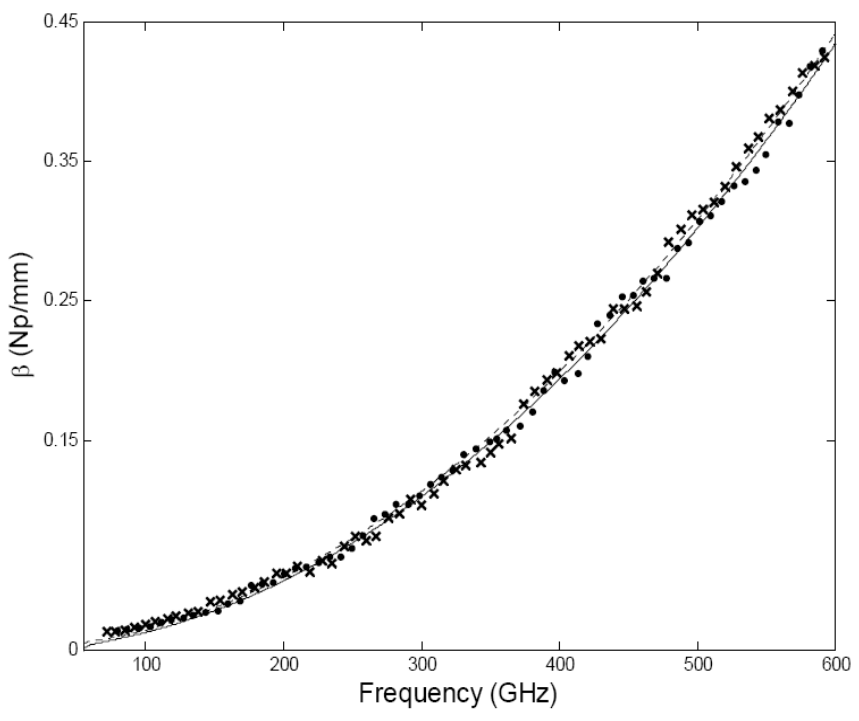

Fig.7. The points in this figure are the measured attenuation coefficients for the FB6H.V material (crosses for the + sense of polarization and dots for the sense).

The forms of these measured spectra of the real and imaginary parts of the refractive indices are to be understood as explained in Section II. The refractive indices of a uniaxial gyrotropic material are related to the material's underlying circularly-polarised transverse permeabilities,

$$
\mu_{ \pm}(\omega) \equiv \mu_{ \pm}^{\prime}(\omega)+i . \mu_{ \pm}^{\prime \prime}(\omega),
$$

and permittivities, $\varepsilon_{ \pm}(\omega) \equiv \varepsilon_{ \pm}^{\prime}(\omega)+i . \varepsilon_{ \pm}^{\prime \prime}(\omega)$, thus $[5,6]$

$$
n_{ \pm}(\omega) \equiv \sqrt{\mu_{ \pm}(\omega) \cdot \varepsilon_{ \pm}(\omega)}
$$

and, if the imaginary parts of $\mu_{ \pm}(\omega)$ and of $\varepsilon_{ \pm}(\omega)$ are small compared with their real parts:

$$
\begin{gathered}
n_{ \pm}^{\prime}(\omega) \square \sqrt{\mu_{ \pm}^{\prime}(\omega) \cdot \varepsilon_{ \pm}^{\prime}(\omega)} \quad \text { and } \\
n_{ \pm}^{\prime \prime}(\omega) / n_{ \pm}^{\prime}(\omega) \square\left\{\mu_{ \pm}^{\prime \prime}(\omega) / \mu_{ \pm}^{\prime}(\omega)\right\}+\left\{\varepsilon_{ \pm}^{\prime \prime}(\omega) / \varepsilon_{ \pm}^{\prime}(\omega)\right\} .
\end{gathered}
$$


The spectra of the measured real parts of the refractive indices shown in Fig. 6 are indicative of magnetic resonance, i.e. a permeability, $\mu_{ \pm}^{\prime}(\omega)$, having the resonance form shown below, and a real part of the permittivity, $\varepsilon^{\prime}(\omega)$, which is common to both senses of polarisation and increases slowly with frequency in this range:

$$
n_{ \pm}^{\prime}(\omega) \square \sqrt{\mu_{ \pm}^{\prime}(\omega) \cdot \varepsilon^{\prime}(\omega)}=\sqrt{1+\frac{\omega_{m}}{\left(\omega_{a}-\omega_{m}\right) \mp \omega}} \cdot \sqrt{\varepsilon^{\prime}(\omega)} .
$$

The following values for $\omega_{a}, \omega_{m}$ and $\varepsilon^{\prime}(\omega)$ in this expression give the fine lines in Fig.6 providing a close fit to the measured values of $n_{ \pm}^{\prime}(\omega)$ :

$$
\begin{gathered}
\omega_{a} / 2 \pi=55.8 \mathrm{GHz} ; \omega_{m} / 2 \pi=10.1 \mathrm{GHz} \\
\varepsilon^{\prime}(\omega)=20.5 \text { at } 100 \mathrm{GHz} \text { to } 20.75 \text { at } 600 \mathrm{GHz} .
\end{gathered}
$$

The spectra of the measured imaginary parts of the refractive indices, $n_{ \pm}^{\prime \prime}(\omega)$ (plotted in Fig. 7 as an attenuation coefficient $\left.\beta(\omega) \equiv(\omega / c) \cdot n^{\prime \prime}(\omega)\right)$ correspond to a $\tan \delta \equiv$ $2 n_{ \pm}^{\prime \prime}(\omega) / n_{ \pm}^{\prime}(\omega)$ that increases proportionally with frequency, from $4.10^{-3}$ at $100 \mathrm{GHz}$ to $16.10^{-3}$ at $600 \mathrm{GHz}$. These values are no more than a few times larger than the values of $\tan \delta$ in this range of frequency for Alumina, a low-loss dielectric ceramic [9]. This promises acceptable insertion losses in devices incorporating this material at frequencies up to at least $400 \mathrm{GHz}$ [2]. As indicated above, $\tan \delta$ has contributions from the imaginary parts of both the permeability and the permittivity; the fact that there is little difference at the higher frequencies between the measured values of $\beta_{ \pm}(\omega)$ for the two senses of circular-polarisation suggests that the losses there are primarily due to dielectric processes, and can be assigned to $\varepsilon_{ \pm}^{\prime \prime}(\omega)$ in this model of the electromagnetic properties of the material. It is not possible separately to identify in the data the several possible contributions to dielectric loss considered in Section II.

We believe the rms deviations of the points in Figures 6 and 7 from smooth curves over an interval of frequency containing several such points provides a fair estimate of the reproducibility inherent in our measurements. Measuring the reproducibility of each point in such measurements by making many repeated runs through the full range of frequency would be a time-consuming task and we have not undertaken that. The dynamic range in the primary recorded data [12] underlie these deviations, of course, but the recorded data through most of a peak or trough in transmittance are sampled when determining the frequency at a maximum or minimum (to give a value for the real part of the refractive index), or when determining the values of transmittance at the maximum or minimum (to give a value for the imaginary part); the separation of the points in frequency in Figures 6 and 7 therefore indicates the resolution interval over which each determination of refractive index applies. The time taken in recording the primary data is pertinent, of course, since the magnitude of random uncertainties should reduce on increasing the recording time. The total recording time for the data used in producing the measurements in Figures 6 and 7 was no more than several minutes. Most of the time taken in making such measurements is spent, not in recording data, but in configuring the transmissometer with the VNA (and we should perhaps have taken more time in recording the data when making the measurements reported here).

We have noted in Section II that values for the reduced waveimpedances, $z_{ \pm}(\omega)$, as well as for the refractive indices, $n_{ \pm}(\omega)$, are required in order to completely specify the magneto-optical characteristics of a uniaxial gyrotropic material. Measured values for $z_{ \pm}(\omega)$ can be obtained from the measured transmittance spectra of a plate-sample because $z_{ \pm}(\omega)$ is related to the single-surface reflectance, $r_{1 \pm}(\omega)$, which appears in the above formulae for transmittances,

$$
z_{ \pm}(\omega) \equiv\left(1+r_{1 \pm}(\omega)\right) /\left(1-r_{1 \pm}(\omega)\right)
$$

The procedure used for determining values for $n_{ \pm}^{\prime \prime}(\omega)$ from the measured transmittances, as outlined above, also provides measures of $r_{1 \pm}(\omega)$ and thence of $z_{ \pm}(\omega)$. The values of $z_{ \pm}(\omega)$ obtained in this way are, however, particularly sensitive to measurement uncertainties in the initial determinations of transmitted power [15] and, though the values obtained suffice for use in designing circulators incorporating the FB6H.V material [2], and though the model introduced above for the permeability and permittivity provides a fit to the measured values within the range of uncertainty, that range of uncertainty in our present determinations does not allow a sensitive test of model, in particular whether the values of $\varepsilon^{\prime}(\omega)$ are in fact the same for the two senses of polarization at these frequencies, and whether some part of the observed losses should be assigned to the imaginary part of the permeability. In order to resolve those questions we are seeking an improved procedure for measuring the single-surface reflectance.

A rather stringent test of the model, and of the rather lengthy overall procedures that we have followed in our determinations of $n_{ \pm}(\omega)$ for this hexaferrite ceramic from the initial measurements of co- and cross-polar transmittances of a plate-sample (including a few approximations), is to start with the model and work back through the formulae to obtain co- and cross-polar transmittances of a plate-sample, and then to compare the results with the initial measurements of these quantities. The results of this test are satisfactory through the frequency range covered, up to $600 \mathrm{GHz}$. As an illustration of this, Figure 8 shows the comparison for the range 250 to 300 GHz. 

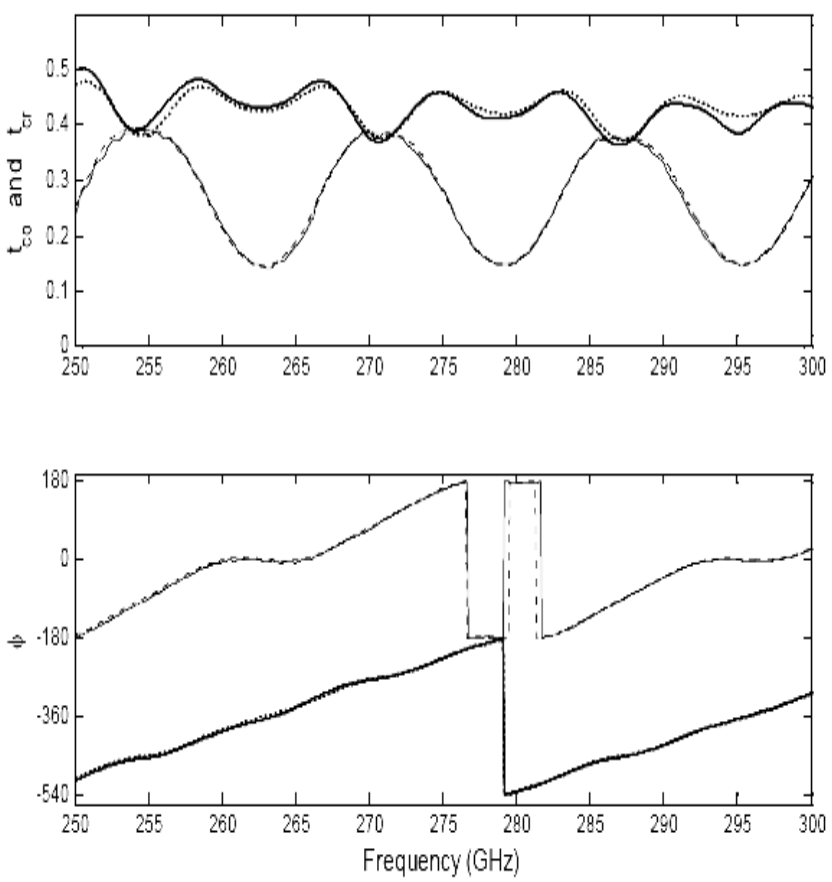

Fig.8 The co- and cross-polar transmittances of the FB6H.V plate, the moduli above and phases below in reduced zones +180 to -180 degrees for the copolar case and -180 to -540 degrees for the cross-polar (to separate the lines); the continuous lines are the initially measured data and the broken lines are simulated from the model (see the text); the bold lines are for the cross-polar case.

\section{CONCLUDING SUMMARY.}

We have demonstrated in Section III that the magneto-optical constants of a hexaferrite ceramic can be determined over a wide range of submillimter-wave frequencies, from 75 to 600 $\mathrm{GHz}$, using a multi-band vector-network-analyser and a wideband quasi-optical transmissometer. Free-space, or quasioptical, transmissometers have previously been used for measuring the magneto-optical constants of ferrite materials over limited ranges of millimeter-wave frequencies [3, 16]. Our measurements cover a much wider, continuous, range of frequency, however, well into the sub-millimeter-wave range, and the methods we use for determining the magneto-optical constants of a material from the measured transmittances of a plate-sample differ from those employed in the measurements made at the lower millimeter-wave frequencies. It has been necessary to design and manufacture wave-guide feed-horn units having very low impedance discontinuities for each of the five wave-guide bands that make up the range from 75 to $600 \mathrm{GHz}$, and efficient quasi-optical components for the transmissometer that cover the full frequency range. The high degree of collimation of the test beams achievable at these higher frequencies reduces the levels of systematic error in the measurements (as demonstrated here by the measurements on plate samples of high-purity monocrystalline silicon).

The results of measurements reported here indicate some success in our objective to demonstrate a method for the rapid assessment of candidate magneto-optical materials over the range 75 to $600 \mathrm{GHz}$ with a precision that will allow the results to be used in the engineering design of circulators incorporating the materials. The need for such a method has increased in the light of the fact that we have found small variations of the magneto-optical constants from one plate of a batch of a high-quality commercially-available hexaferrite ceramic to another; though small these variations would be significant in the engineering design of a circulator for this range of frequency, which means that measurements of the magneto-optical constants will have to be made on each sample plate that is to be used in a circulator. These frequency-domain methods provide higher scan-speeds and frequency-resolutions than the time-domain techniques that have been the most widely-used methods for spectrometric measurements in this range of frequency [12].

A notable finding of our measurements is that, though most grain-oriented hexaferrite ceramics show marked signal-beam attenuation in the $\mathrm{mm} / \mathrm{submm}$-wave range, a particular grainoriented hexaferrite ceramic (TDK strontium hexaferrite FB6H) exhibits relatively low submillimeter-wave attenuations, decidedly less than ten times the attenuations in high-quality dielectric ceramics such as Alumina in this range of frequency. There is some prospect that supplementary processing of this material will further reduce the attenuation, a matter that warrants further investigation.

A further notable finding of our measurements is that a selected grain-oriented hexaferrite ceramic has magnetooptical constants consistent with a simple magnetic-resonance model up to more than ten times the resonance frequency, in spite of the complex structure characteristic of a ceramic material (which structure is essential for the required permanent-magnet properties). This promises wide-band circulators for use well into the submillimeter-wave range. The measurements we have reported here have, in fact, already been used in the design and manufacture of highperformance wideband circulators that are now in use in a number of major measurement systems at frequencies up to $350 \mathrm{GHz}$ [2].

\section{ACKNOWLEDGMENTS}

We thank H. Taguchi and K.Suzuki of the TDK Corporation for supplying modified versions of one of their standard ferrite products. Bin Yang wishes to express his appreciation of the invaluable guidance he received from John Dupuy of the Department of Electronic Engineering, Queen Mary University of London, in the educated use of vector-networkanalysers.

\section{REFERENCES}

[1] V.G.Harris et. al., "Recent advances in processing and applications of microwave ferrites”, J. Magn. Magn. Mater. 321, pp. 2035-2047, 2009.

[2] D.H.Martin and R.J.Wylde, "Wideband circulators for use at frequencies above $100 \mathrm{GHz}$ to beyond $350 \mathrm{GHz}$ ", IEEE Trans. Microw. Theory Tech, vol 57, no. 1, pp. 99-108, Jan. 2009.

[3] K. N. Kocharyan, M. N. Afsar and I. I. Tkachov "New Method for Measurements of Complex Magnetic Permeability in the Millimetre- 
Wave Range, Part II: Hexaferrites”, IEEE Trans. Magnetics. vol. 35, No.4 July 1999, pp 2104-2110.

[4] The web pages of the TDK Corporation, Tokyo, Japan.

[5] K. J. Standley, Oxide Magnetic Materials, Clarendon Press Oxford, 1972. P.J.B. Clarricoats, Microwave Ferrites, Chapman and Hall, 1961.

[6] D. H. Martin, "The gyrotropic characteristics of hexaferrite ceramics" available at http://www.elec.qmul.ac.uk/people/robert/publications.htm

[7] J.A.Stratton, Electromagnetic Theory, McGraw-Hill, 1941, p. 511. M.Born and E.Wolf, Principles of Optics, Sixth Edition, Pergamon Press, 1980.

[8] D.H.Martin, "The study of the vibrations of crystal lattices by far-infrared spectroscopy”, Advances in Physics, vol. 14, pp 39- 99, 1965.

[9] P.F.Goldsmith, Quasioptical Systems, IEEE Press/Chapman \& Hall, 1998. D.H.Martin and J.W.Bowen, "Long-Wave Optics," IEEE Trans. Microw. Theory Tech., vol. 41, pp.1676-1690, Oct 1993.

[10] The web pages of Thomas Keating Ltd., Billingshurst, UK.

[11] B.Lax, J.A.Weiss, N.W.Harris and G.F.Dionne, "Quasi-optical Ferrite Reflection Circulator”, IEEE Trans. Microw. Theory Tech. vol. 41, pp2190-2197, Dec. 1993.

[12] The web pages of AB Millimetre, Paris, France.

[13] M. N. Afsar and Hua Chi, "Millimetre-wave complex dielectric permittivity and loss tangent of extra high purity and compensated silicon”, Int J. Infrared and Millimeter Waves, vol. 15, no. 7, 1994.

[14] J. R. Birch et. al., "An intercomparison of measurement techniques for the determination of the dielectric properties of solids at near-millimeter wavelengths”, IEEE Trans. Microw Theory Tech.,vol 42, pp. 956-965, June 1994.

[15]Bin Yang, D.H.Martin, R.S.Donnan, R..J.Wylde, "Quasi-optical measurements of Magnetic Materials at Frequencies up to $630 \mathrm{GHz}$," The Joint $33^{\text {rd }}$ international Conference on Infrared and Millimetre Waves and $16^{\text {th }}$ International Conference on Terahertz Electronics (IRMMWTHz2008), Sep 15-19, 2008, in California Institute of Technology, Pasadena, California USA.

[16]A.C.Lynch et al, "Free-wave measurement of permeability and permittivity of ferrites at millimeter-wave frequencies", IEE Proc.Sci.Meas.Technol, vol 142, March 1995.
Ph.D degrees in Electronic Engineering in 2004 and 2008 respectively, in the Antenna and Electromagnetics Group of the Department of Electronic Engineering, Queen Mary, University of London, U.K., where he is currently a post-doctoral assistant engaged in the development of $\mathrm{mm} / \mathrm{submm}$-wave and $\mathrm{THz}$ measurement systems.

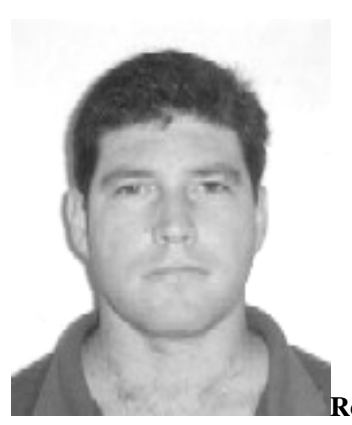
Physics) in 2000 from the University of Wollongong, NSW, Australia and became a post-doctoral research assistant in the Department of Electronic Engineering at Queen Mary, University of London, U.K. in 2001, developing diffracted Gaussian beam optics for the design-verification of quasi-optical systems. In 2003 he was appointed to a Lectureship in the Department and is engaged in the development of $\mathrm{mm} / \mathrm{submm}$-wave and $\mathrm{THz}$ measurement systems for basic and applied research into medical and structural imaging and spectrometric studies of materials, metamaterials and biomaterials.

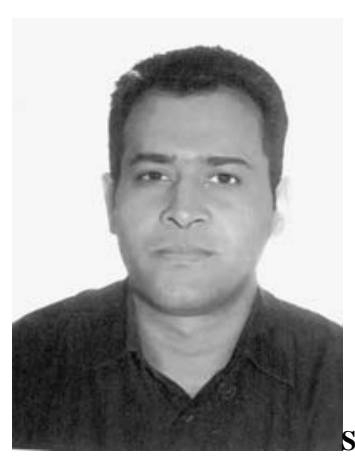
Degree (Microwaves and Radio communications) in 1998 from the Conservatoire National des Arts et Métiers (CNAM), Paris, France. He is currently working as RF Engineer Responsible of the manufacture, development and testing of Millimeter Vector Network Analyzers (MVNA 8$1000 \mathrm{GHz}$ and accessories) at AB Millimetre SAS Paris FRANCE.

For photographs and biographies for $\mathbf{R} \mathbf{J}$ Wylde and $\mathbf{D} \mathbf{H}$ Martin see IEEE Trans. MTT, vol. 57, no. 1, Jan. 2009, and for P Goy see IEEE Trans. MTT, vol. 55, no. 5, May 2007.

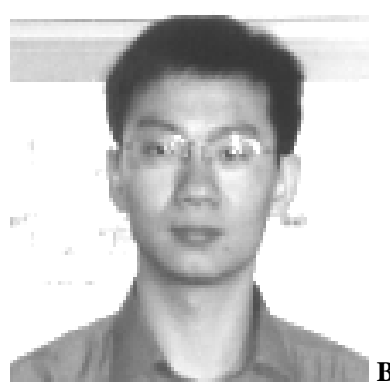
of Posts and Telecommunications, China, in 2001. He received his MSc and 